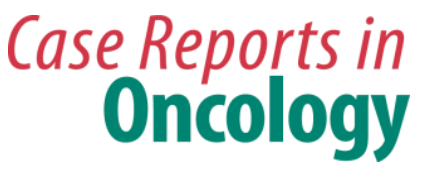

Case Rep Oncol 2018;11:252-257

DOI: $10.1159 / 000488669$

Published online: May 2, 2018

Published by S. Karger AG, Base

www.karger.com/cro

This article is licensed under the Creative Commons Attribution-NonCommercial 4.0 International License (CC BY-NC) (http://www.karger.com/Services/OpenAccessLicense).

Usage and distribution for commercial purposes requires written permission.

\title{
Second-Line Treatment of HER2-Positive Salivary Gland Tumor: Ado-Trastuzumab Emtansine (T-DM1) after Progression on Trastuzumab
}

\author{
Tatiana Strava Corrêa ${ }^{a} \quad$ Gustavo Duarte Ramos Matos $^{b} \quad$ Marcos Segura $^{c}$ \\ Carlos Henrique dos Anjos ${ }^{a}$ \\ ${ }^{a}$ Division of Medical Oncology, Hospital Sírio-Libanês, Brasilia, Brazil; ${ }^{b}$ Division of Medical \\ Oncology, Instituto do Cancer do Estado de São Paulo, São Paulo, Brazil; 'Division of \\ Medical Pathology, Lamina Pathology Laboratory, Brasilia, Brazil
}

\section{Keywords}

Trastuzumab emtansine $\cdot$ Salivary gland tumor $\cdot$ HER2 positivity $\cdot$ Second line

\begin{abstract}
Patients with salivary duct cancer (SDC) and HER2 overexpression could receive trastuzumab in combination with chemotherapy for metastatic disease. No standard treatment exists for patients with HER2-positive metastatic SDC after progression. We report an excellent patient response to second-line treatment with T-DM1 after progression on paclitaxel plus trastuzumab. Case Report: In June 2014, a 79-year-old male patient underwent right parotidectomy and ipsilateral radical neck dissection after the diagnosis of parotid carcinoma. Pathological staging demonstrated locally advanced disease with the involvement of 13 lymph nodes (levels I, II, III, and IV), with extracapsular extravasation. He underwent adjuvant radiotherapy ending in December 2014. A PET scan in March 2015 diagnosed recurrent and systemic disease, with bone lesions, neck lymph node involvement, and hepatic metastasis. The immunohistochemistry showed HER2 receptor overexpression $(+3 /+3)$. The patient received first-line trastuzumab plus paclitaxel beginning in April 2015. After 6 cycles, his response was confirmed by PET scan. In February 2016, he had symptoms of disease progression, and a PET
\end{abstract}


scan revealed disease progression in the neck, bones, and liver. He started T-DM1 in April 2016. The neck skin lesions disappeared after 6 cycles, with low toxicity. PET scans performed every 3 months showed response in the liver and bone lesions. Conclusions: We report the case of a patient with SDC treated with T-DM1, with a very good response. Salivary carcinoma is a rare disease for which no randomized clinical trials are available. The maintenance of HER2 blockage might be important in this disease.

(C) 2018 The Author(s)

Published by S. Karger AG, Basel

\section{Introduction}

Malignant salivary gland tumors are a rare type of head and neck cancer, accounting for up to $11 \%$ of all head and neck cancers. The majority occur in the parotid gland. They often exhibit aggressive behavior with a propensity to recur and metastasize. Metastases to regional lymph nodes indicate poor prognosis and are associated with reduced survival. In $20 \%$ of cases, distant organ metastasis can occur, usually in the lungs, bone, or other visceral organs $[1,2]$.

Surgery is the main treatment for resectable disease, and radiation is also considered for most patients after surgery. Systemic chemotherapy for metastatic cases often has disappointing results [3].

Salivary gland tumors exhibit HER2 overexpression with an incidence of up to $20 \%$ in mucoepidermoid carcinomas and an even higher proportion (30-40\%) in salivary duct cancer (SDC) $[4,5]$. The overexpression of HER2 is a factor in poor prognosis and is associated with more aggressive tumors [6]. The HER2 proto-oncogene, which encodes a membrane receptor protein of the epidermal growth factor receptor family, is overexpressed in a variety of epithelial malignancies [7]. Targeting the HER2 pathway in cancers such as breast cancer and gastric adenocarcinoma has already been investigated, and there is a group of different drugs designed to block this pathway. They include monoclonal antibodies (trastuzumab, pertuzumab), tyrosine kinase inhibitors (lapatinib, neratinib), and an antibody-drug conjugate consisting of the monoclonal antibody trastuzumab linked to the cytotoxic agent emtansine (T-DM1) [8, 9].

Some case series and reports have demonstrated the benefit of combined chemotherapy and trastuzumab for patients with metastatic SDC [9]. After progression, there is no standard treatment for these patients. We report the case of a patient with excellent response to second-line treatment with T-DM1 after progression on paclitaxel plus trastuzumab.

\section{Case Report}

In June 2014, a 79-year-old male patient underwent right parotidectomy and ipsilateral modified radical neck dissection after the diagnosis of a parotid carcinoma. Pathological staging demonstrated high-grade salivary duct carcinoma and locally advanced disease with the involvement of 13 lymph nodes at levels I, II, III, and IV, with extracapsular extravasation. As an adjuvant treatment, he underwent radiotherapy, ending in December 2014.

A PET scan performed in March 2015 diagnosed local recurrence and systemic disease involving the neck lymph nodes, bone, and hepatic metastasis. At that time, we conducted immunohistochemistry (IHC) of the primary lesion, which demonstrated HER2 receptor overexpression (+3/+3) (Fig. 1, Fig. 2). 
As a first-line treatment, the patient received trastuzumab plus paclitaxel from April 2015 until February 2016. After the first 6 cycles, the new PET scan showed excellent partial response. The treatment was changed to trastuzumab alone, but after 3 months, the disease progressed. Then, he was rechallenged with paclitaxel plus the antibody. In February 2016, the patient experienced symptoms of progressive disease such as fatigue and cervical pain. A PET scan revealed progressive disease in the neck (local), bones, and liver. Because the expression of androgen receptors was observed in the salivary tumor, he started treatment with bicalutamide from February until April 2016. He underwent a rapid worsening of symptoms, weight loss, and neck skin progression, and scans showed progression in all lesions.

He started T-DM1 in April 2016. The skin lesions on the neck disappeared after 6 cycles. PET scans were performed every 3 months and showed response in the liver and bone lesions. The performance status and symptoms improved. In March 2017, 1 year after the TDM1 response, the patient presented worsening of the neck skin lesions with local bleeding, suggesting disease progression. A new scan showed stable disease in the liver but progressive disease in the bones and neck (skin). The next treatment was combined vinorelbine with trastuzumab plus pertuzumab. Again, he showed a clinical response (neck lesions), and PET scans showed decrease in the SUV of the hepatic lesions until July 2017.

The patient developed chronic dysphagia due to actinic esophagitis, leading to aspiration pneumonia and the need for the passage of an enteral catheter for feeding in August 2017, when he started exclusive supportive care. He was in home care when he passed away in September 2017, 2.5 years after the diagnosis of a metastatic salivary gland tumor.

\section{Discussion}

Salivary gland malignant neoplasms have a variety of histologic subtypes with varying expression levels of HER2, both in immunohistochemical assays and FISH. Usually, cancers of intercalated duct origin, such as adenoid cystic carcinoma and acinic cell carcinoma, consist of low-grade disease with indolent behavior, in contrast with disease derived from the secretory ducts, such as salivary duct carcinoma and mucoepidermoid carcinoma, which tend to have a more aggressive course [1,2]. A previous study found that the frequency of HER2 is higher in those aggressive subtypes, and in one of them, the frequency of HER2 positivity found by IHC reached $83 \%$ in salivary duct carcinoma, $14 \%$ in adenocarcinoma, and $21 \%$ in mucoepidermoid carcinoma [2-4].

HER2 expression is associated with poor prognosis according to various series of cases examining cancer-specific mortality and time to relapse, independently of the histology in which it occurred [5-7].

Currently, there are no treatment guidelines for systemic therapy for salivary gland tumors. As SDC is rare, we lack randomized studies to determine the real impact of trastuzumab in these patients. Some phase II trials, case series, and case reports have demonstrated benefits for patients treated with combined chemotherapy and trastuzumab after metastasis. After progression, there is no standard treatment for patients with HER2positive metastatic SDC [8-11].

A phase II study was conducted including incurable disease with tumors found to be HER2 positive by IHC, but it was prematurely closed due to insufficient recruitment. However, it documented a very prolonged response, lasting more than 2 years [12]. 
T-DM1 is a monoclonal antibody-cytotoxic drug conjugate combining trastuzumab with emtansine, a microtubule inhibitor. The EMILIA trial showed the superiority of this compound over lapatinib plus capecitabine after progression on trastuzumab in HER2-overexpressing breast cancer, and T-DM1 is currently approved in this scenario [13]. The maintenance of a good performance status after progression on trastuzumab and paclitaxel motivated a trial with T-DM1 in this case.

Even after progression on trastuzumab and T-DM1, the patient still showed response to HER2-targeted therapy when treated with a combination of pertuzumab, trastuzumab, and vinorelbine [14].

A phase I study [15] of neratinib in combination with temsirolimus in patients with HER2-dependent solid tumors included one patient with SDC. The best response of this SDC was stable disease after progression on trastuzumab. A recent case report describes a patient with refractory metastatic HER2-positive SDC with progressive cerebellar metastases who obtained a transient clinical benefit from treatment with neratinib, a HER2 tyrosine kinase inhibitor [16].

Beyond HER2-targeted therapy, the SDC showed the expression of androgen receptors [17]. In this case, the patient experienced disease progression on bicalutamide.

This case reports a patient with salivary carcinoma treated with T-DM1. Because of the rarity of disease, there are no randomized clinical trials, so treatment is guided by what we know of breast cancer with HER2 overexpression. As good response to this treatment has been observed, other patients with salivary carcinoma with HER2 expression should also maintain HER2 blockage even after first-line progression. In addition, routine testing for HER2 expression in newly diagnosed metastatic salivary gland cancers is recommended.

\section{Acknowledgements}

We thank the "Instituto de Ensino e Pesquisa" of the Hospital Sirio-Libanês for the support in the article review.

\section{Statement of Ethics}

We obtained the patient's informed consent.

\section{Disclosure Statement}

The authors have no conflicts of interest to declare.

\section{References}

1 Weed DT, Gomez-Fernandez C, Pacheco J, Ruiz J, Hamilton-Nelson K, Arnold DJ et al. MUC4 and ERBB2 expression in major and minor salivary gland mucoepidermoid carcinoma. Head Neck. 2004 Apr;26(4):35364.

2 Glisson B, Colevas AD, Haddad R, Krane J, El-Naggar A, Kies M et al. HER2 expression in salivary gland carcinomas: dependence on histological subtype. Clin Cancer Res. 2004 Feb;10(3):944-6.

3 Jaehne M, Roeser K, Jaekel T, Schepers JD, Albert N, Löning T. Clinical and immunohistologic typing of salivary duct carcinoma: a report of 50 cases. Cancer. 2005 Jun;103(12):2526-33. 
4 Skálová A, Stárek I, Vanecek T, Kucerová V, Plank L, Szépe P et al. Expression of HER-2/neu gene and protein in salivary duct carcinomas of parotid gland as revealed by fluorescence in-situ hybridization and immunohistochemistry. Histopathology. 2003 Apr;42(4):348-56.

5 Cornolti G, Ungari M, Morassi ML, Facchetti F, Rossi E, Lombardi D et al. Amplification and overexpression of HER2/neu gene and HER2/neu protein in salivary duct carcinoma of the parotid gland. Arch Otolaryngol Head Neck Surg. 2007 Oct;133(10):1031-6.

6 Press MF, Pike MC, Hung G, Zhou JY, Ma Y, George J et al. Amplification and overexpression of HER-2/neu in carcinomas of the salivary gland: correlation with poor prognosis. Cancer Res. 1994 Nov;54(21):5675-82.

7 Nakano T, Yamamoto H, Hashimoto K, Tamiya S, Shiratsuchi H, Nakashima T et al. HER2 and EGFR gene copy number alterations are predominant in high-grade salivary mucoepidermoid carcinoma irrespective of MAML2 fusion status. Histopathology. 2013 Sep;63(3):378-92.

8 Alotaibi AM, Alqarni MA, Alnobi A, Tarakji B. Human epidermal growth factor receptor 2 (HER2/neu) in salivary gland carcinomas: a review of literature. J Clin Diagn Res. 2015 Feb;9(2):ZE04-08.

9 Thorpe LM, Schrock AB, Erlich RL, Miller VA, Knost J, Le-Lindqwister N et al. Significant and durable clinical benefit from trastuzumab in 2 patients with HER2-amplified salivary gland cancer and a review of the literature. Head Neck. 2017 Mar;39(3):E40-4.

10 Iqbal MS, Shaikh G, Chatterjee S, Cocks H, Kovarik J. Maintenance therapy with trastuzumab in her2 positive metastatic parotid ductal adenocarcinoma. Case Rep Oncol Med. 2014;2014:162534.

11 Ghazali N, Parker L, Settle K, Lubek JE. Sustained response of HER2-positive metastatic salivary adenocarcinoma, not otherwise specified, treated with trastuzumab. Oral Surg Oral Med Oral Pathol Oral Radiol. 2016 Sep;122(3):292-9.

12 Haddad R, Colevas AD, Krane JF, Cooper D, Glisson B, Amrein PC et al. Herceptin in patients with advanced or metastatic salivary gland carcinomas. A phase II study. Oral Oncol. 2003 Oct;39(7):724-7.

13 Verma S, Miles D, Gianni L, Krop IE, Welslau M, Baselga J et al.; EMILIA Study Group. Trastuzumab emtansine for HER2-positive advanced breast cancer. N Engl J Med. 2012 Nov;367(19):1783-91.

14 Baselga J, Cortés J, Kim SB, Im SA, Hegg R, Im YH et al.; CLEOPATRA Study Group. Pertuzumab plus trastuzumab plus docetaxel for metastatic breast cancer. N Engl J Med. 2012 Jan;366(2):109-19.

15 Gandhi L, Bahleda R, Tolaney SM, Kwak EL, Cleary JM, Pandya SS et al. Phase I study of neratinib in combination with temsirolimus in patients with human epidermal growth factor receptor 2-dependent and other solid tumors. J Clin Oncol. 2014 Jan;32(2):68-75.

16 Sorenson KR, Piovezani Ramos G, Villasboas Bisneto JC, Price K. Targeted approaches applied to uncommon diseases: a case of salivary duct carcinoma metastatic to the brain treated with the multikinase inhibitor neratinib. Case Rep Oncol. 2017 Aug;10(2):726-31.

17 Yamamoto N, Minami S, Fujii M. Clinicopathologic study of salivary duct carcinoma and the efficacy of androgen deprivation therapy. Am J Otolaryngol. 2014 Nov-Dec;35(6):731-5. 


\section{Case Reports in Oncology}

\begin{tabular}{l}
\hline Case Rep Oncol 2018;11:252-257 \\
\begin{tabular}{l|l}
\hline DOl: 10.1159/000488669 & ○ 2018 The Author(s). Published by S. Karger AG, Basel
\end{tabular}
\end{tabular} www.karger.com/cro

Corrêa et al: Second-Line Treatment of HER2-Positive Salivary Gland Tumor: Ado-Trastuzumab Emtansine (T-DM1) after Progression on Trastuzumab

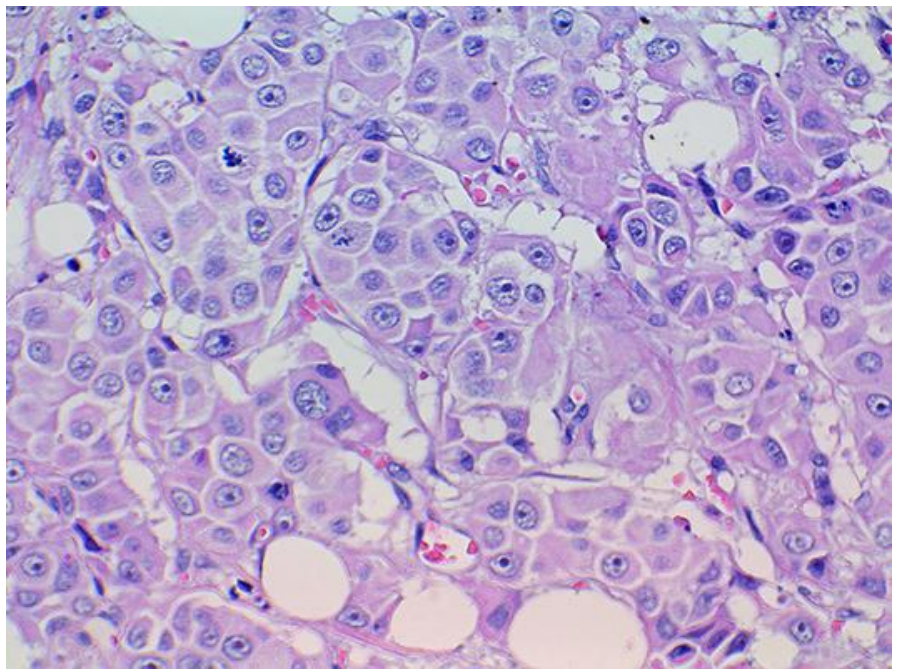

Fig. 1. High-grade salivary duct carcinoma (hematoxylin and eosin).

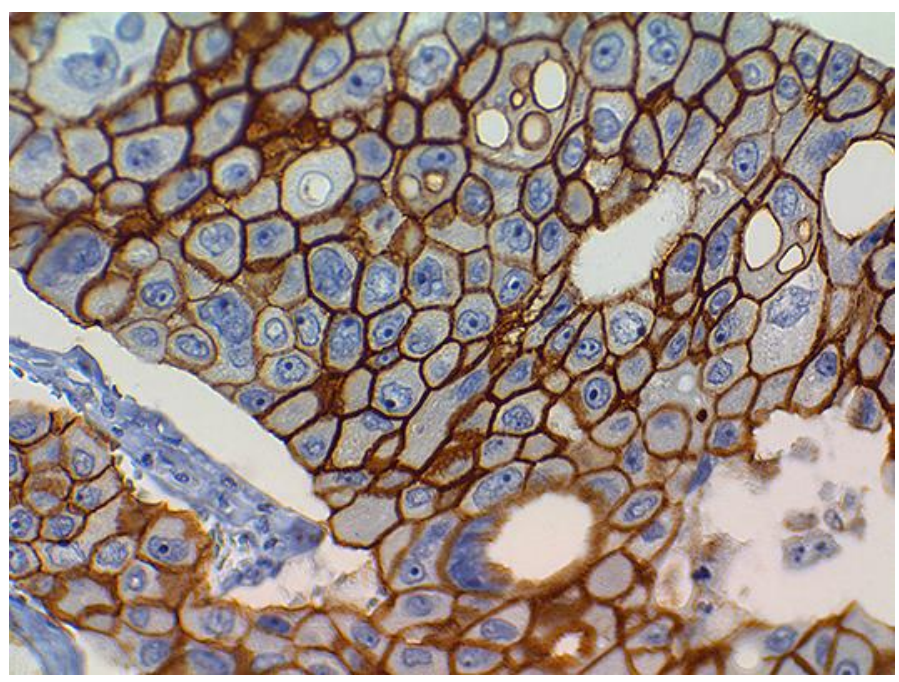

Fig. 2. High-grade salivary duct carcinoma (hematoxylin and eosin). Complete, strong, membranous staining, compatible with 3+ HER2 (ASCO/CAP HER2 expression criteria). 